\title{
Spatially Resolved Far Ultraviolet Spectroscopy of the Jovian Aurora
}

\author{
J. Gustin, D. Grodent, and J. C. Gérard \\ Laboratoire de Physique Atmosphérique et Planétaire, Université de Liège, Liège, Belgium \\ E-mail: gustin@astro.ulg.ac.be
}

and

J. T. Clarke

Department of Astronomy and Center for Space Physics, Boston University, 725 Commonwealth Avenue, Boston, Massachussetts 02215

Received July 9, 2001; revised October 31, 2001

Spatially resolved spectra in four 50- $\AA$ FUV spectral windows were obtained across the jovian aurora with the Space Telescope Imaging Spectrograph (STIS) on board the Hubble Space Telescope. Nearly simultaneous ultraviolet imaging makes it possible to correlate the intensity variations along the STIS slit with those observed in the images and to characterize the global auroral context prevailing at the time of the observations. Spectra at $\sim 1$ - $\AA$ resolution taken in pairs included an unabsorbed window and a spectral region affected by hydrocarbon absorption. Both sets of spectra correspond to an aurora with a main oval brightness of about 130 kilorayleighs of $\mathrm{H}_{2}$ emission. The far ultraviolet color ratios $I(1550-1620 \AA) / I(1230-1300 \AA)$ are 2.3 and 5.9 for the noon and morning sectors of the main oval, respectively. We use an interactive model coupling the energy degradation of incoming energetic electrons, auroral temperature and composition, and synthetic $\mathrm{H}_{2}$ spectra to fit the intensity distribution of the $\mathrm{H}_{2}$ lines. It is found that the model best fitting globally the spectra has a soft energy component in addition to a $10 \mathrm{erg} \mathrm{cm} \mathrm{cm}^{-2} \mathrm{~s}^{-1}$ flux of $80 \mathrm{keV}$ electrons. It provides an effective $\mathrm{H}_{2}$ temperature of $540 \mathrm{~K}$. The relative intensity of temperature-sensitive $\mathrm{H}_{2}$ lines indicates differences between the auroral main oval and polar cap emissions. The amount of methane absorption across the polar region is shown to vary in a way consistent with temperature. For the second spectral pair, the polar cap shows a higher attenuation by $\mathrm{CH}_{4}$, indicating a harder precipitation along high-latitude magnetic field lines. (c) 2002 Elsevier Science (USA) tions.

Key Words: aurora; Jupiter; spectroscopy; ultraviolet observa-

\section{INTRODUCTION}

The jovian far ultraviolet (FUV) auroral spectrum is dominated by the H Lyman- $\alpha$ (Ly $\alpha)$ line and the $\mathrm{H}_{2} \operatorname{Lyman}\left(B{ }^{1} \Sigma_{\mathrm{g}}^{+} \rightarrow\right.$ $\left.X^{1} \Sigma_{\mathrm{g}}^{+}\right)$and Werner $\left(\mathrm{C}^{1} \Pi_{\mathrm{u}} \rightarrow X^{1} \Sigma_{\mathrm{g}}^{+}\right)$band systems between 875 and $1600 \AA$. Low-resolution spectra were obtained first with the Voyager ultraviolet spectrometer (UVS) experiments and later with the International Ultraviolet Explorer (IUE) spec- trograph. The Werner and Lyman bands are predominantly produced by direct excitation of the $\mathrm{H}_{2}$ ground-state molecules with some cascade contribution from higher lying Rydberg singlet states. Low-resolution auroral spectra between 1130 and $1700 \AA$ collected with the Galileo UVS were analyzed with synthetic spectra including absorption by all important hydrocarbons (Ajello et al. 1998, Pryor et al. in press). Observations of the jovian ultraviolet aurora with the Goddard High-Resolution Spectrograph (GHRS) on board the Hubble Space Telescope (HST) have been used to characterize the interaction of the auroral electrons with the jovian atmosphere. Medium-resolution $(\sim 0.5 \AA)$ spectra obtained with the G140M grating mode of the GHRS-HST spectrograph covering a spectral range of $35 \AA$ were analyzed by Clarke et al. (1994), Trafton et al. (1994, 1998), and Kim et al. (1997) to derive rovibrational $\mathrm{H}_{2}$ temperatures. They found rotational temperatures in the range 300$900 \mathrm{~K}$. These values are lower than the $\mathrm{H}_{3}^{+}$rotational (Drossart et al. 1993, Lam et al. 1997) temperatures that probe an ionospheric region at higher altitudes. These inferred $\mathrm{H}_{2}$ temperatures are higher than those derived from Voyager UVS stellar absorption at midlatitudes but are in agreement with the analysis of the Voyager IRIS auroral data by Drossart et al. (1993), as discussed by Grodent et al. (2001).

The $\mathrm{H}_{2}$ FUV color ratio $\mathrm{C}=I(1550-1620 \AA) / I(1230-$ $1300 \AA$ ) (Yung et al. 1982) is used as a measure of the attenuation of the $\mathrm{H}_{2}$ emission by methane and other hydrocarbons overlying the emission layer. It is directly related to the drop in the $\mathrm{CH}_{4}$ absorption cross section at wavelengths below $1350 \AA$ A, which leaves the bulk of the long-wavelength $\mathrm{H}_{2}$ Lyman bands unabsorbed, while the short-wavelength Lyman and Werner bands are absorbed by methane. The color ratio depends on the vertical distribution of the emission and may be used as an indicator of the relative penetration of the electrons into the hydrocarbon layer (Livengood et al. 1990, Harris et al. 1996, Gérard et al. 1998). The color ratios measured in different $35-\AA$ GHRS spectral bands were used by Trafton et al. (1994) and Kim et al. (1997) to derive effective $\mathrm{CH}_{4}$ columns in the range 
$1-7 \times 10^{16} \mathrm{~cm}^{-2}$. The methane columns derived from the Galileo UVS spectra varied from 0 to $10 \times 10^{16} \mathrm{~cm}^{-2}$.

Dols et al. (2000) analyzed seven auroral GHRS spectra, covering the $1220-1750 \AA$ region with $\sim 5$ - $\AA$ resolution obtained in conjunction with the Wide Field Planetary Camera 2 (WFPC2) FUV images. The $1.74 \times 1.74 \operatorname{arcsec}^{2}$ GHRS aperture was moved to different latitudes and local times in both the north and south aurora. In contrast to previous studies, they considered the vertical dimension of the aurora, including temperature, composition, and emission variations with altitude. A primary electron energy spectrum was injected at the top of the prescribed atmospheric model, and the $\mathrm{H}_{2}$ volume emission rate and absorption by hydrocarbons were calculated for each layer of the model. The calculated emerging synthetic $\mathrm{H}_{2}$ spectrum was then compared with the measured $\mathrm{H}_{2}$ intensity distribution to derive the ultraviolet color ratio and the associated electron energy. Large variations of the methane slant column abundance from 2.3 to $70 \times 10^{16} \mathrm{~cm}^{-2}$ were found, reflecting both the changes of the view angle and variations of the characteristic electron energy between 17 and $40 \mathrm{keV}$. The quality of the fits was improved by including a $\mathrm{C}_{2} \mathrm{H}_{2}$ abundance of 0.01 to 0.1 relative to $\mathrm{CH}_{4}$. High-resolution $(\sim 0.07 \AA$ ) Echelle spectra near $1218 \AA$ obtained on the same HST orbit as the medium-resolution spectra yielded a higher $\mathrm{H}_{2}$ temperature than predicted from the model that best reproduced the observed color ratio. This discrepancy was interpreted as an indication that the jovian atmosphere is a few hundred $\mathrm{K}$ warmer than the low-latitude P,T model derived by Seiff et al. (1998) from the Galileo probe in the north equatorial belt. A similar conclusion was reached by Perry et al. (1999) from auroral comparison of the amount of methane absorption and the $\mathrm{H}_{2}$ temperature derived from GHRS spectra.

To further investigate this question, we describe in this study a set of four spatially resolved spectra obtained with the Space Telescope Imaging Spectrograph (STIS) at $\sim 1$ - $\AA$ resolution in conjunction with STIS FUV images. Two pairs of spectra, one absorbed by methane, the other unabsorbed, are compared in order to simultaneously derive the FUV color ratio and the $\mathrm{H}_{2}$ rovibrational temperature. A new modeling approach is introduced based on the self-consistent model by Grodent et al. (2001) which calculates the vertical temperature and composition structure as well as the emerging $\mathrm{H}_{2}$ spectrum consistent with a prescribed electron energy flux at the top of the atmo- sphere. In this scheme, the only adjustable parameters are the characteristic energies and fluxes of auroral electrons producing the FUV aurora. This model is applied to determine the characteristic electron energy derived from the $\mathrm{H}_{2}$ rovibrational distribution and the FUV color ratio.

\section{OBSERVATIONS AND DATA REDUCTION}

Far ultraviolet observations of Jupiter were obtained in August 1999 with the STIS on board the HST. We used both the imaging and spectroscopic capabilities of STIS to obtain (a) spatially resolved FUV spectra at medium spectral resolution and (b) images using the FUV multianode microchannel array (MAMA) detector. The MAMA detector is a $1024 \times 1024$ photon counting detector providing a two-dimensional far ultraviolet image. It can be operated either in the ACCUM mode to give a timeintegrated image or in the TIMETAG mode to produce an event stream with fast time resolution. Two sets of images and two spectra were taken during two consecutive HST orbits. The first image in each orbit was obtained in the ACCUM mode with the clear filter while the second one was taken in the TIMETAG mode using the $\mathrm{SrF}_{2}$ filter which rejects the atomic hydrogen Ly $\alpha$ emission. The resulting passband is centered at $1469 \AA$ and covers the 1280-1780 A range. The two images taken in each orbit were followed by two spectra using the G140M grating with the $52 \times 0.5 \operatorname{arcsec}^{2}$ slit, covering an $\sim 50$ - $\AA$ wavelength range and a spectral resolution close to $1 \AA$. All observation times are listed in Tables Ia and Ib, together with the exposure length, operating mode, bandwidth, central wavelength, central meridian longitude (CML), and coordinates of the central element.

We mainly used the flat fielded science ( $f t t)$ data in this study, corrected for the detector sensitivity. The spectroscopic $f t t$ file gives a linear wavelength scale in the dispersion direction and a uniform sampling in the spatial direction while the imaging $f t$ provides the final calibrated product. One of the steps of the pipeline reduction process consists in removing the dark signal (count rate in the detector in the absence of photons from the sky). The dark files used in most past STIS data reduction processes did not take into account the time evolution of the mean dark rate. Landsman (1998) correlated the mean dark rate and the MAMA low-voltage power supply temperature (LVPST) to quantify the rate changes. At low temperature, the dark rate is spatially uniform with a constant rate of about $6.2 \times 10^{-6}$ count

TABLE Ia

FUV-MAMA G140M $52 \times 0.5$ Arcsec Spectra

\begin{tabular}{lccccccccc}
\hline File ID & Fig. ID & $\begin{array}{c}\text { Date } \\
\text { (start) }\end{array}$ & $\begin{array}{c}\text { UT } \\
\text { (startpoint) }\end{array}$ & $\begin{array}{c}\text { Exposure } \\
(\mathrm{s})\end{array}$ & Mode & $\begin{array}{c}\text { Bandwidth } \\
(\AA)\end{array}$ & $\begin{array}{c}\text { Central } \\
\text { wavelength }(\AA)\end{array}$ & $\begin{array}{c}\text { CML } \\
\text { (deg) }\end{array}$ & $\begin{array}{c}\text { Pointing } \\
(\text { lat, long.) (deg) }\end{array}$ \\
\hline o5hy02twq & 4a & $08 / 13 / 99$ & $14: 08: 50$ & 620 & TIMETAG & 56 & 1222 & 138.4 \\
o5hy02tyq & 4b & $08 / 13 / 99$ & $14: 24: 51$ & 620 & TIMETAG & 56 & 1567 & 148.0 & 54,148 \\
o5hy02u4q & 4c & $08 / 13 / 99$ & $15: 44: 35$ & 640 & TIMETAG & 56 & 1321 & 196.2 & 45,199 \\
o5hy02u6q & $4 d$ & $08 / 13 / 99$ & $16: 00: 56$ & 640 & TIMETAG & 56 & 1518 & 206.1 & 45,197 \\
\hline
\end{tabular}


TABLE Ib

FUV-MAMA Images

\begin{tabular}{lcccccccr}
\hline File ID & $\begin{array}{c}\text { Date } \\
\text { (start) }\end{array}$ & $\begin{array}{c}\text { UT } \\
\text { (startpoint) }\end{array}$ & $\begin{array}{c}\text { Exposure } \\
(\mathrm{s})\end{array}$ & Mode & Filter & $\begin{array}{c}\text { Bandwidth } \\
(\AA)\end{array}$ & $\begin{array}{c}\text { Central wavelength } \\
(\AA)\end{array}$ & $\begin{array}{c}\text { CML } \\
(\text { deg })\end{array}$ \\
\hline 05hy02tuq & $08 / 13 / 99$ & $13: 57: 39$ & 300 & TIMETAG & $\mathrm{SrF}_{2}$ & 120 & 1469 & 131.6 \\
o5hy02u2q & $08 / 13 / 99$ & $15: 33: 24$ & 300 & TIMETAG & $\mathrm{SrF}_{2}$ & 120 & 1469 & 189.5 \\
\hline
\end{tabular}

pixel $^{-1} \mathrm{~s}^{-1}$, while above a LVPST temperature of $38.9^{\circ} \mathrm{C}$, the dark rate increases exponentially with temperature to reach a maximum dark rate of about $5.0 \times 10^{-5}$ count pixel ${ }^{-1} \mathrm{~s}^{-1}$. This increase mainly occurs in a "blotch" area in the upper left portion of the detector. As seen in Fig. 1, the auroral part of the spectrum is located in the lower portion of the detector. However, the "blotch" effect is not negligible (also visible in Fig. 1) and it is necessary to carefully account for its presence, especially in this study in which some portions of the spectra have low count rates.

In addition, comparisons of dark observations obtained since the beginning of the STIS operations indicate that the "blotch" evolves in time, in terms of count rate but also in spatial extension (Fig. 2). Because of this evolution of the "blotch," the statistical relationship provided by Landsman (1998) is not accurate enough to properly subtract the dark component. This temperature dependence is based on a dark file which is a coaddition of 199 dark files leading to a total exposure time of $272,820 \mathrm{~s}$ (the "superdark" file). In that method, the LVPST is a parameter used to determine the scaling factor to apply to the "superdark" in order to subtract the "blotch." The shortcoming of this procedure is that the "superdark" is a mean of dark exposures taken in different periods with different temperatures: the normalized "superdark" applied to the data will not represent the situation of the detector at the time of our specific observations because of this count rate variability. To remove the dark count rate from the data, we used a dark exposure taken three days after our observations, the closest calibration observation in time during this period (Table II). Consequently, starting from the pipeline $f t$ file, we applied the following procedure: (i) add to the science file the mean dark value that was removed during the pipeline reduction process; (ii) normalize the appropriate dark file to the exposure time of the observations; (iii) smooth the dark matrix to remove the high frequencies due to noise; and (iv) subtract the resulting dark from the data. The variation of intensity along the slit is used to test our method, assuming that the distribution of the airglow intensity along the slit is approximately flat as predicted over this limited range of solar zenith angles and view

TABLE II

MAMA FUV Dark Used to Reduce the Data

\begin{tabular}{lccccc}
\hline File ID & $\begin{array}{c}\text { Date } \\
(\text { start })\end{array}$ & $\begin{array}{c}\text { UT } \\
\text { (startpoint) }\end{array}$ & $\begin{array}{c}\text { Length } \\
(\mathrm{s})\end{array}$ & Mode & Filter \\
\hline o5iioqclq & $08 / 16 / 99$ & $16: 52: 34$ & 1380 & ACCUM & Long_Pass \\
\hline
\end{tabular}

angles. As shown in Fig. 3, our corrections yield a fairly constant signal after the dark noise subtraction.

Three spectra were extracted from each file, using the UV intensity distribution along the slit as a criterion: the first covers the limb region of the jovian disk; the second corresponds to the main auroral oval; the third includes the polar cap region. As seen in Fig. 1, each region is characterized by a specific spectrally integrated intensity signature. The region outside the planetary disk does not show any specific spatial feature but it is used to subtract the auroral spectra of any geocoronal contribution. The limb region is very intense and is characterized by a narrow peak; the polar cap gives a diffuse distribution over a larger extent. The main oval shows a well defined but less intense peak than the limb. Finally, the midlatitude airglow region does not show any spatial structure, as it is excited by $\mathrm{H}_{2}$ fluorescence and photoelectron impact with a contribution from ultraviolet reflected sunlight at longer wavelengths. Figure 3 shows the spectrally integrated intensity variation along the STIS slit extracted from the 1320 - $\AA$ spectrum. This spectrum is the most sensitive to the temperature-dependent dark noise, owing to its low signal-to-noise ratio. Figure 4 shows the extracted spectra for the two orbits. The total intensity of the three spectra of each exposure is normalized to the same value in the spectral window. Although differences are observed in the relative brightness of some individual lines, the overall shape of each spectrum is similar for the three spectra. To define the global auroral morphology at the time of the spectral observations, we used the two time-tagged images integrated over the exposure time. These images are closer in time to the spectra and their high-pass filter eliminates the Ly $\alpha$ contribution, which may have a different spatial distribution from the $\mathrm{H}_{2}$ bands measured with the MAMA clear mode.

\section{ATMOSPHERIC MODEL AND SYNTHETIC SPECTRA}

\section{III.1. Atmospheric Model}

The interactive one-dimensional model developed by Grodent et al. (2001) has been used to generate $\mathrm{H}_{2}$ synthetic spectra consistent with the prescribed auroral precipitation. This model couples a two-stream electron transport model of energy deposition with a 1-D thermal conduction model including thermal cooling to calculate a self-consistent thermal structure adapted to the auroral upper atmosphere. The two-stream code calculates the flow and redistribution of the electron energy as a function 


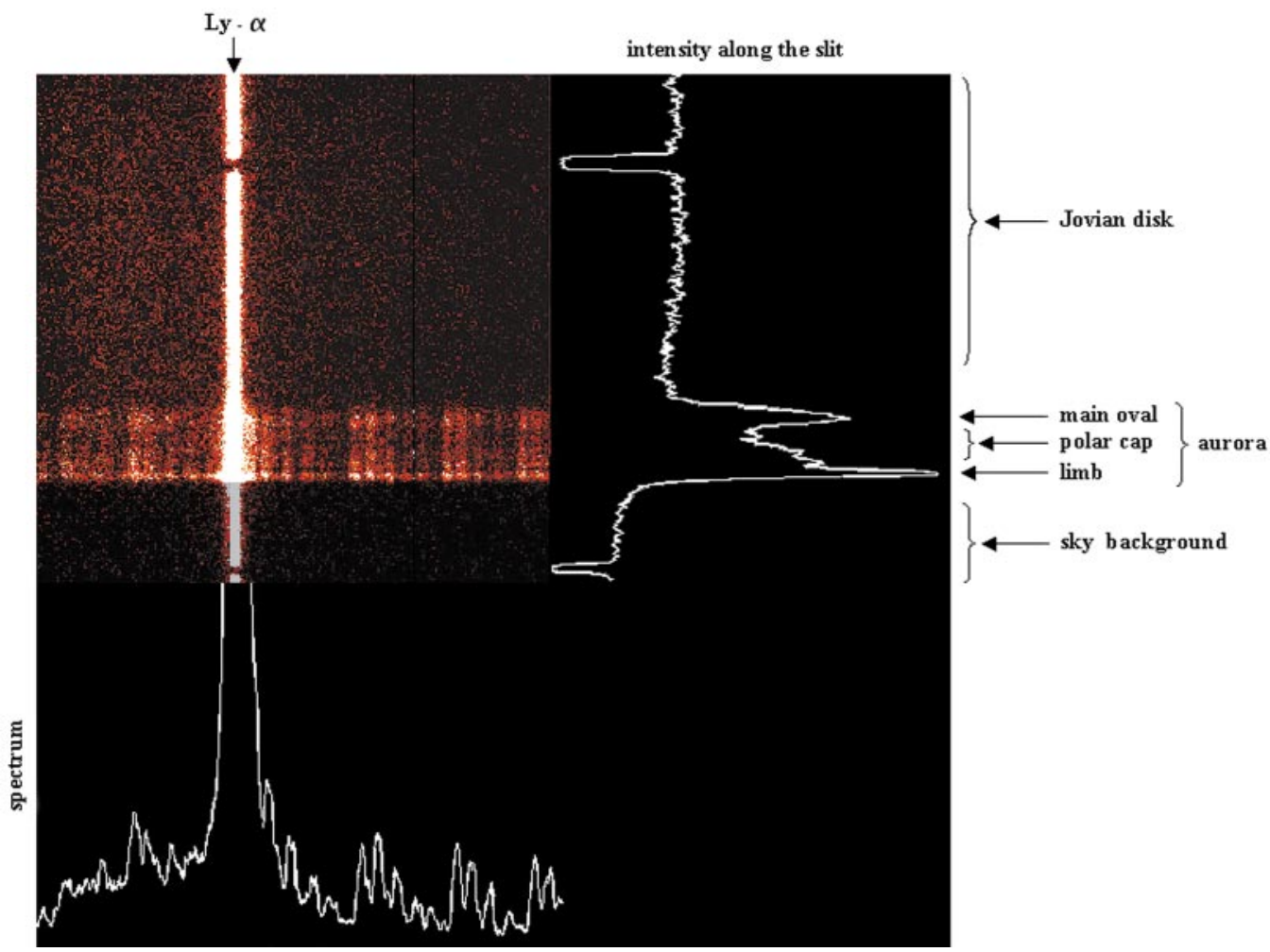

FIG. 1. Count distribution on the MAMA detector. A spectrum in a particular spatial region is obtained by summing a selected number of lines while a sum over a selected number of columns gives the variation of intensity along the slit in a given spectral window.

of altitude from atomic and molecular processes by means of discrete energy bins. Once the electron fluxes are calculated, the rates of ionization, dissociation, heating and emission processes can be determined. To account for the effects of the auroral electron precipitation on the thermal structure of the upper atmosphere, we consider the balance between heating, cooling, and heat transport by thermal conduction. Four major heat sources are considered: (a) chemical heating initiated by

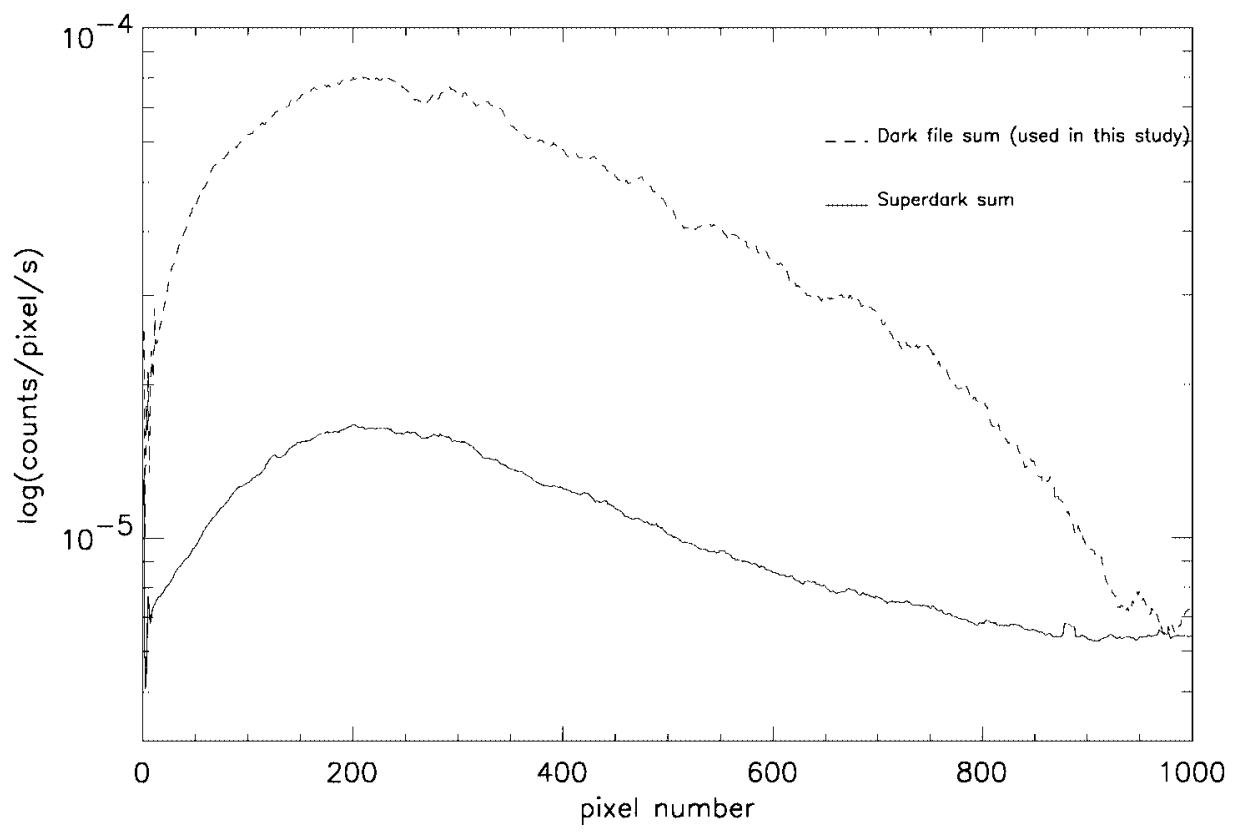

FIG. 2. Dark file "o5iioqclq" summed over the 1024 lines of the detector used to subtract the "blotch" from the data. The same sum for superdark file "mama_fuvdark.fits" is shown for comparison. It clearly illustrates the change of the dark background in amplitude and spatial location. 

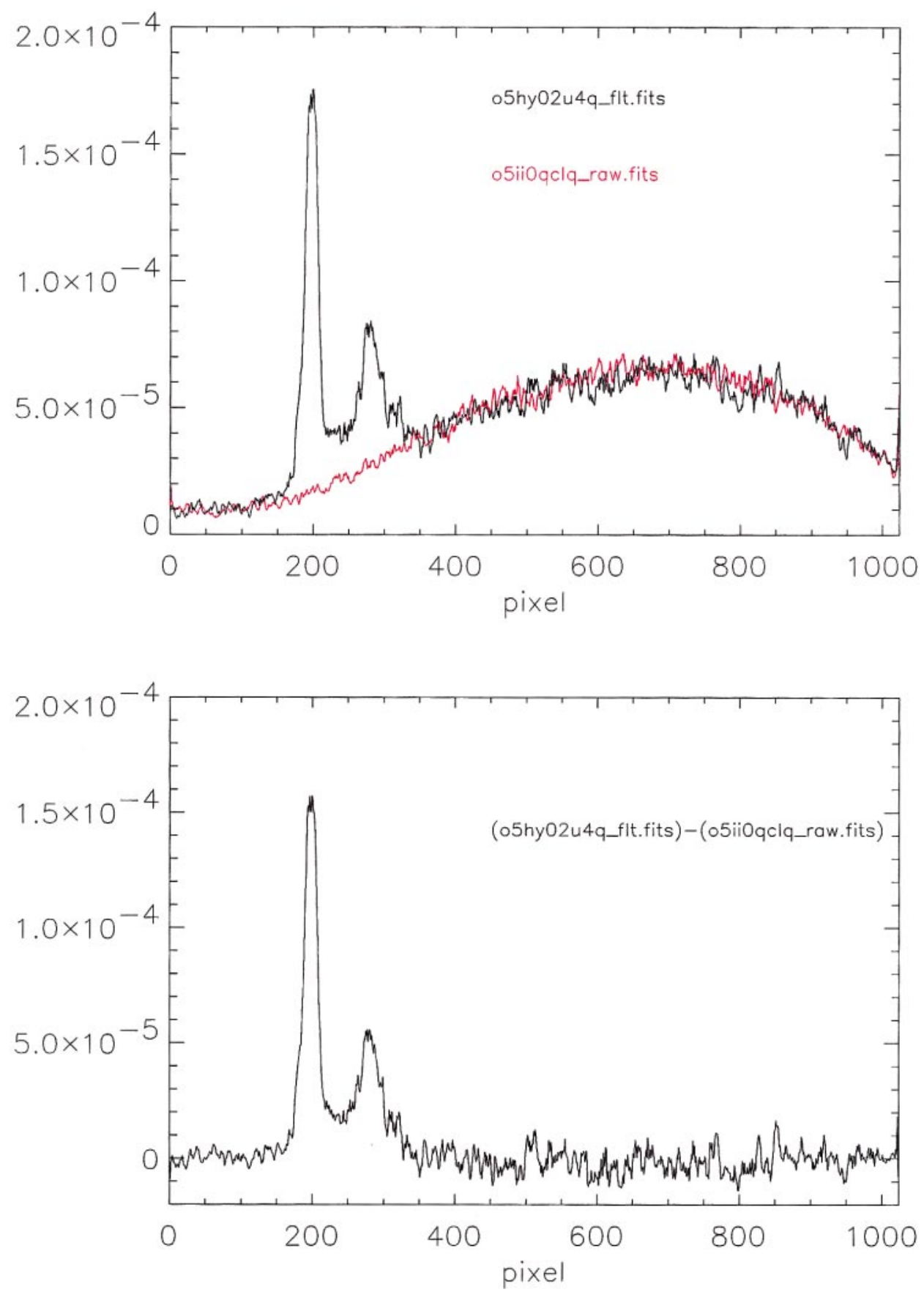

FIG. 3. Integrated intensity along the slit for the second absorbed spectrum, plotted with the integrated intensity along the slit for the dark file (top). The same spectrum with the dark count contribution subtracted. The shape of the integrated intensity of the airglow region is flat, as expected (bottom).

the ionization of $\mathrm{H}_{2}$ and $\mathrm{H}$, (b) excess kinetic energy resulting from $\mathrm{H}_{2}$ dissociation, (c) vibrational excitation of the ground electronic state of $\mathrm{H}_{2}$ following Lyman and Werner band transitions, and (d) thermal electron cooling of energetic electrons, and subsequent $\mathrm{H}_{2}$ vibrational and rotational cooling of the thermal electrons. Altitude-dependent radiative cooling by thermal infrared band emissions (assuming cooling to space approximation) from $\mathrm{H}_{3}^{+}, \mathrm{CH}_{4}$, and $\mathrm{C}_{2} \mathrm{H}_{2}$ is considered to be the major heat sink. In order to self consistently account for the adjustment of the densities to the new temperature profile, the code iteratively solves the diffusion equations (assuming an equilibrium between mixing and diffusive equilibrium for $\mathrm{H}_{2}, \mathrm{H}, \mathrm{He}, \mathrm{CH}_{4}$, and $\mathrm{C}_{2} \mathrm{H}_{2}$ ). The initial atmosphere is based on an analytical fit of the thermal profile determined by Seiff et al. (1998) from the Galileo probe data and hydrocarbon mixing ratios from Gladstone et al. (1996). Altitudes are calculated from pressures and temperatures by integrating the hydrostatic equilibrium equation. The reference altitude $(0 \mathrm{~km})$ corresponds 

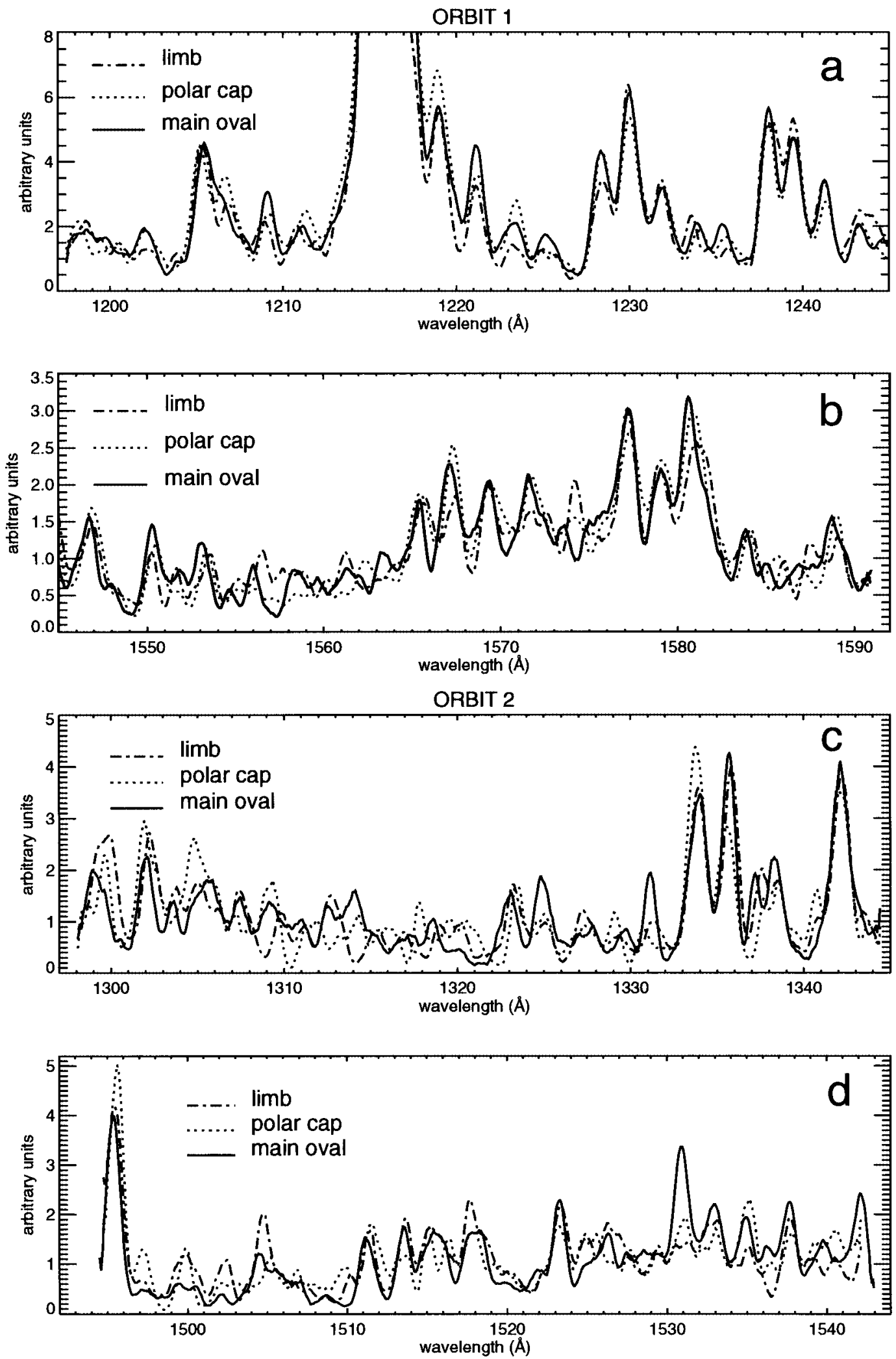

FIG. 4. Plots of the four STIS observations in the three regions of the aurora, smoothed over $0.5 \AA$. The three spectra are normalized to the same total intensity in the spectral window. 
to a pressure level of 1 bar and accounts for the value of the acceleration of gravity at $60^{\circ} \mathrm{N}$ at thermospheric altitudes. The $\mathrm{H}_{3}^{+}$density is estimated assuming photochemical equilibrium, although in the upper thermosphere the characteristic transport becomes shorter than the chemical lifetime.

We have considered an energy distribution of the electrons giving rise to a discrete aurora, that is, an aurora with a peak altitude near $245 \mathrm{~km}$, compatible with the $\mathrm{H}_{2}$ visible emission observed by Galileo (Vasavada et al. 1999). We use a Maxwellian distribution for the high-energy component of the distribution. Additional low-energy electrons (hereafter referred to as soft electrons) have been added to heat the upper atmosphere and produce the extreme ultraviolet (EUV) emission observed with the Hopkins Ultraviolet Telescope (HUT) (Wolven and Feldman 1998) and the Galileo Extreme Ultraviolet Spectrometer (Ajello et al. 2001), as discussed by Grodent et al. (2001). They are represented by an additional Maxwellian component. The initial energy flux distribution thus has the form

$$
\Phi(E)=A \Phi_{0,1} \frac{E}{E_{0,1}} \exp \left(-\frac{E}{E_{0,1}}\right)+B \Phi_{0,2} \frac{E}{E_{0,2}} \exp \left(-\frac{E}{E_{0,2}}\right)
$$

where $A$ and $B$ are normalization factors, $\phi_{0,1}$ and $E_{0,1}$ are the energy flux and the characteristic energy of the high-energy Maxwellian distribution, and $\phi_{0,2}$ and $E_{0,2}$ are the energy fluxes and the characteristic energies associated with the Maxwellian soft electron energy distribution. They are determined based on the spectra, using both the $\mathrm{H}_{2}$ temperature and the color ratio information.

\section{III.2. Coupled Atmosphere-Spectral Model}

The synthetic spectral generator described in detail in Dols et al. (2000) is coupled with the one-dimensional model defined above to fit the observed spectra. The jovian upper atmosphere is divided into 20 layers equally spaced in $\log _{10} \mathrm{P}$ coordinates and extending from $5.4 \times 10^{-5}$ to $4.6 \times 10^{-14}$ bar. A local synthetic spectrum is generated for each layer and uses the main outputs of the 1-D model (electron energy distribution, $\mathrm{H}_{2}$ temperature and density, and $\mathrm{CH}_{4}$ and $\mathrm{C}_{2} \mathrm{H}_{2}$ density). The final emerging spectrum which is used to fit the data is the weighted sum of the 20 spectra, including absorption effects when present and smoothing by the instrumental line spread function. The resolution of the observed spectra $(\sim 1 \AA)$ allows the identification of lines whose relative intensity strongly depends on temperature as well as lines partially absorbed by acetylene and methane (the "unabsorbed" spectra are slightly absorbed by $\mathrm{C}_{2} \mathrm{H}_{2}$ ). The temperature-dependent line intensities are an indicator of the mean rovibrational $\mathrm{H}_{2}$ temperature, i.e., the temperature of each layer of the upper atmosphere weighted by the volume emission rate. The relative brightness of the lines absorbed by $\mathrm{C}_{2} \mathrm{H}_{2}$ and the first spectrum of each observation pair absorbed by $\mathrm{CH}_{4}$ vary with the hydrocarbon profile as well as the altitude of the emission peak. In this analysis, we assume that the $\mathrm{H}_{2}$ temperature and the color ratio are unchanged between the two observations of each orbit. Series of model atmospheres and synthetic spectra were generated for various combinations of characteristic energies of the initial electron distribution. The fit giving the best compromise between temperature, color ratio, $\mathrm{C}_{2} \mathrm{H}_{2}$ absorption, and the minimum $\chi^{2}$ with the observed spectrum is considered the best fit.

\section{ANALYSIS}

\section{IV.1. First Orbit: August 13, 1999, 13:51-14:35}

We first examine the data set collected during the first HST orbit. A FUV image was taken close in time before the spectra and allows the superposition of the projection of the $52 \times 0.5 \operatorname{arcsec}^{2}$ slit used for the spectra on the image. Pointing of the telescope was achieved by using the blind offset method, which introduces an uncertainty of up to 1 arcsec in absolute position and adds to the shift due to the guide star reacquisition between the images and spectra. The images taken with the $1024 \times 1024$ pixel detector subtend a field of view of $24.7 \times 24.7 \mathrm{arcsec}^{2}$. Therefore, the 0.5 arcsec width of the spectral slit represents approximately 20 pixels on the image. We first extract the portion of the image corresponding to the slit of the spectrograph. This emission was summed over the 20 pixel width and over 20 pixels along the 1024 pixel length. The resulting intensity distribution was compared with the integrated intensity along the slit of the second spectrum of the first orbit (1490-1540 ̊). The first spectrum was not used for this comparison since its spectral range lies outside the bandpass of the image. We allow the location of the slit center to be displaced by up to 1 arcsec until a good match is obtained between the position of the auroral bright features seen in the image and the spectrum. As seen in Fig. 5, the agreement is good and the relative magnitude of the different peaks is
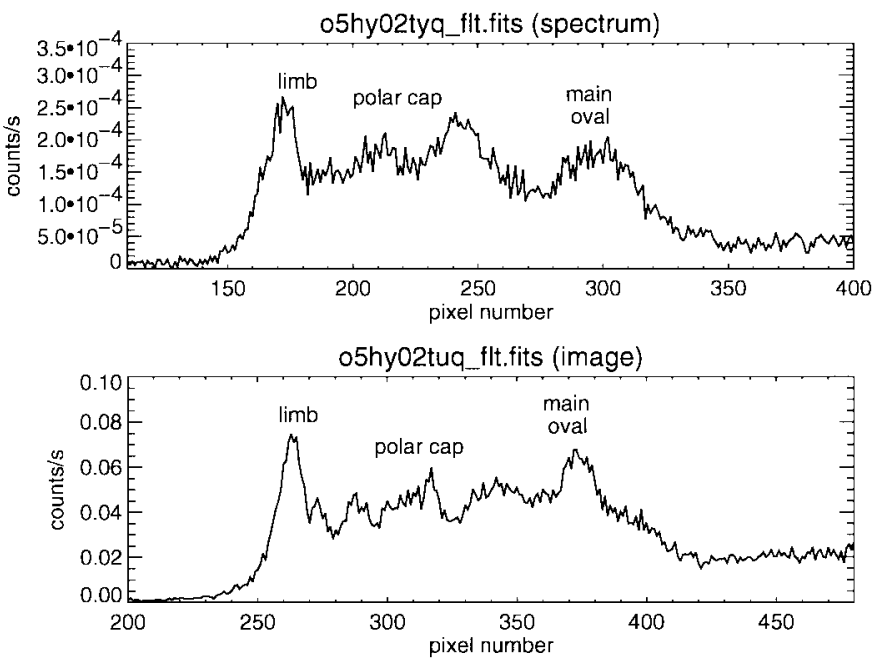

FIG. 5. Comparative plots of the integrated brightness along the slit for the first unabsorbed spectrum and for the image. The similarity between the two curves was used to offset the slit from the center of the image within 1 arcsec. Three auroral regions can be clearly identified as the limb, polar cap, and main oval. 


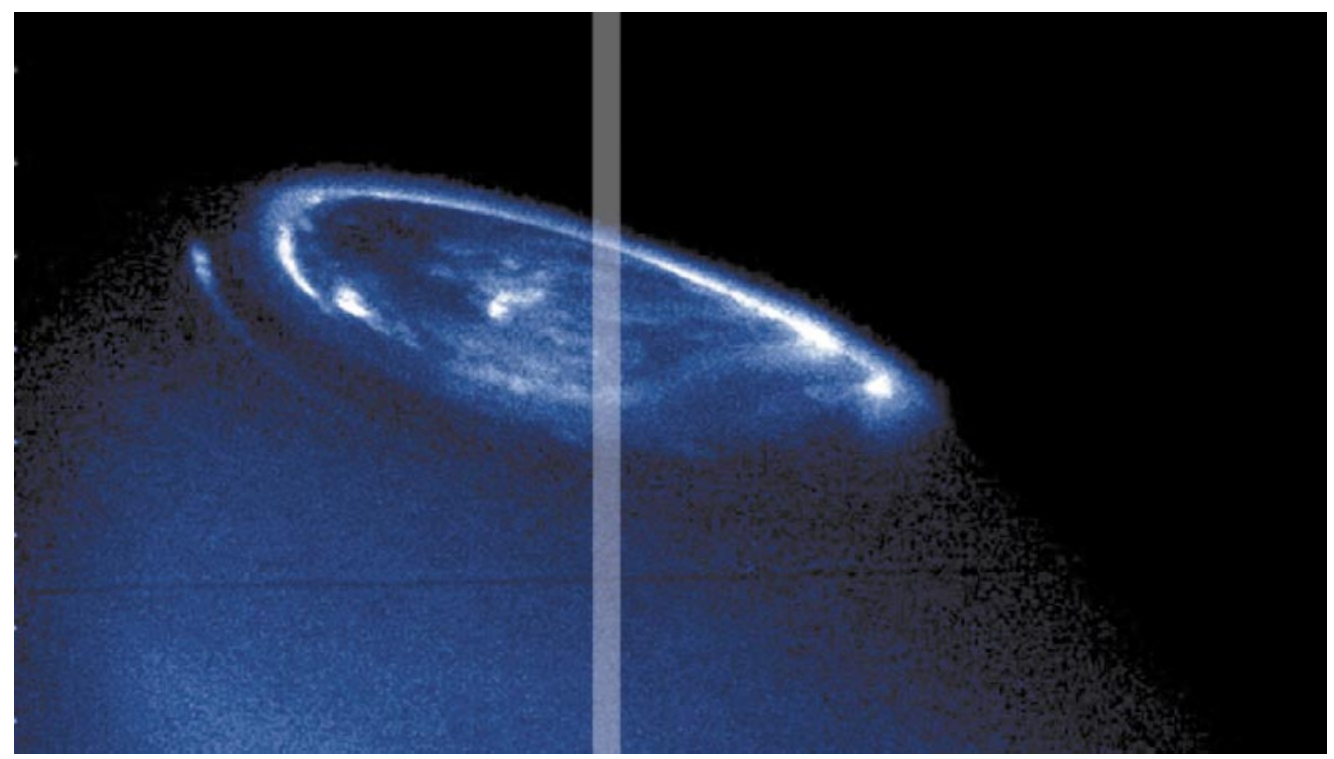

FIG. 6. Projection of the slit on the image taken on the first orbit. The total $\mathrm{H}_{2}$ brightness of the aurora was derived from the image and is comparable (within $10 \%$ ) to the spectrum $\mathrm{H}_{2}$ brightness.

preserved. Some differences occur, probably because of possible temporal variations and because the bandpasses do not exactly overlap. Figure 6 shows the $52 \times 0.5 \operatorname{arcsec}^{2}$ slit on the image as well as the limb, polar cap, and main oval regions.

Since the overall shapes of the spectra extracted from the three auroral regions are quite similar, the analysis first focuses on the spectra extracted from the main auroral oval. The first spectrum (1194-1250 ), smoothed over $0.5 \AA$, is plotted in physical units in Fig. 7. It is dominated by the Ly $\alpha$ line at $1215.7 \AA$. The intensity integrated over the spectral window, excluding Ly $\alpha$, is about 9 kilo-rayleighs. The second spectrum (1540-1595 ̊) was taken

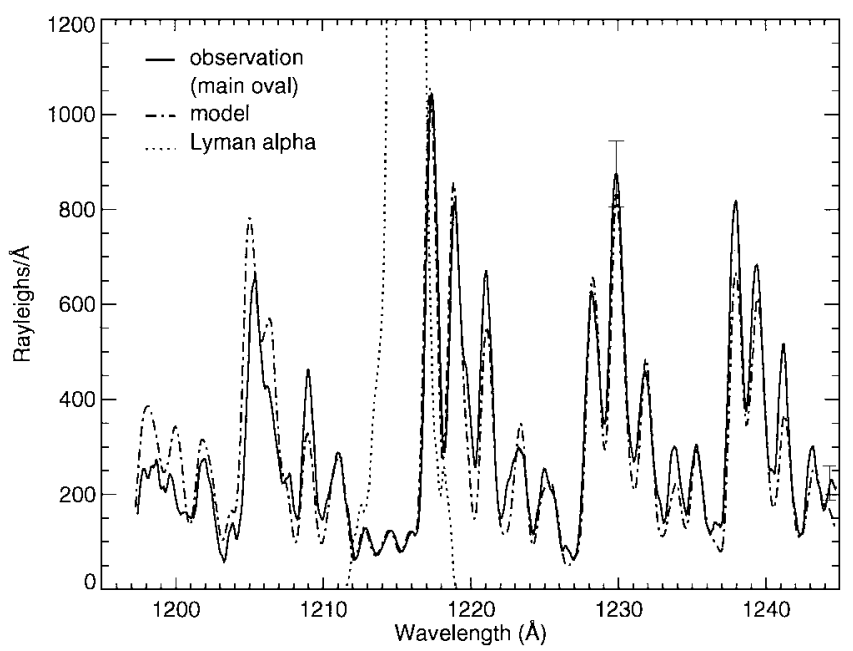

FIG. 7. Comparison between the first absorbed spectrum of the main oval and the best model fit. The Ly $\alpha$ contribution was removed from the data to fit the $\mathrm{H}_{2}$ spectrum and restored afterwards. One $\sigma$ error bar is shown for two of the lines.
$15 \mathrm{~min}$ later and is characterized by an integrated intensity of about 11 kilo-rayleighs (Fig. 8). Since the first spectrum is partly absorbed by methane while the second one is not, it is possible to derive a color ratio $R=I(1550-1620 \AA) / I(1230-1300 \AA)$ from these spectra. It should be noted that the intensities are expressed in rayleighs, so that $R$ is 1.25 times the original color ratio defined by Yung et al. (1982), expressed in energy units. Since the bandwidths of the first pair of observed spectra are 1194$1250 \AA$ and $1540-1595 \AA$, respectively, we used an unabsorbed synthetic spectrum to extrapolate their total intensities to the bandpasses relevant for $R$. The numerator of $R$ has been directly estimated from the second (unabsorbed) observed spectrum, using a synthetic unattenuated spectrum. The denominator of $R$

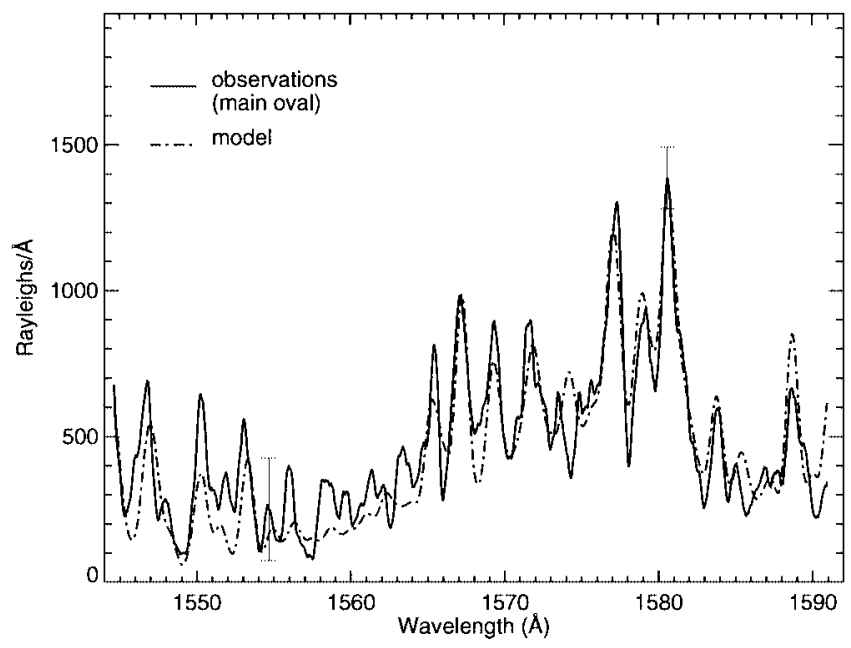

FIG. 8. First unabsorbed spectrum and comparison with the best model fit. 
cannot be estimated as simply from the first (absorbed) spectrum because in the 1194-1250- $\AA$ range, the wavelength dependence of the $\mathrm{CH}_{4}$ absorption cross section must be taken into account. The 1194-1204 and 1225-1250- $\AA$ regions were analyzed separately to avoid the contribution of the bright Ly $\alpha$ line. Accordingly, we estimated the $\mathrm{CH}_{4}$ column responsible for the attenuation of the 1225-1250- $\AA$ spectrum. More specifically, one can write

$$
\begin{aligned}
& I_{(1225-1250)}^{\mathrm{obs}}=I_{(1225-1250)}^{\mathrm{u}} \exp \left(-1 \sigma_{(1225-1250)}\right)=\alpha I_{(1225-1250)}^{\mathrm{ufit}} \\
& I_{(1490-1540)}^{\mathrm{obs}}=I_{(1490-1540)}^{\mathrm{u}}=\beta I_{(1490-1540)}^{\mathrm{ufit}},
\end{aligned}
$$

where $I_{\mathrm{w}}^{\mathrm{obs}}$ is the observed spectrum in the spectral window w, $I^{\mathrm{u}} \mathrm{W}$ is the unabsorbed synthetic spectrum in the same spectral window, $I_{\mathrm{w}}^{\text {ufit }}$ is the synthetic spectrum used to fit the observed spectrum in the region $\mathrm{w}, \alpha$ and $\beta$ are the scaling factors applied to the synthetic spectrum to best fit the data, $\sigma_{\mathrm{w}}$ is the mean methane cross section in the w spectral window, and 1 is the effective column of methane overlying the auroral emission providing the observed color ratio. Dividing the two Eqs. (1) side by side, one gets

$$
l=\frac{1}{\sigma_{(1225-1250)}} \ln \left(\frac{\beta}{\alpha}\right)
$$

where it is assumed that the $I_{(1490-1540)}^{\text {ufit }} / I_{(1225-1250)}^{\text {ufit }}$ ratio is equivalent to the $I_{(1490-1590)}^{\mathrm{u}} / I_{(1225-1250)}^{\mathrm{u}}$ ratio. Accounting for the wavelength dependence of the methane absorption cross section gives the relations

$$
\begin{aligned}
& I_{(1230-1300)}^{\mathrm{obs}}=I_{(1230-1300)}^{\mathrm{u}} \exp \left(-1 \sigma_{(1230-1300)}\right) \\
& I_{(1225-1250)}^{\mathrm{obs}}=I_{(1225-1250)}^{\mathrm{u}} \exp \left(-1 \sigma_{(1225-1250)}\right),
\end{aligned}
$$

where $I_{(1230-1300)}^{\mathrm{obs}}$ is, by extrapolation, the spectrum (the denominator of $R$ ) that would have been observed in that range. It is expressed in terms of the observed intensity $I_{(1225-1250)}^{\text {obs }}$ by dividing Eqs. (3) side by side:

$$
\begin{aligned}
I_{(1230-1300)}^{\mathrm{obs}}= & \frac{I_{(1230-1300)}^{\mathrm{u}}}{I_{(1225-1250)}^{\mathrm{u}}} I_{(1225-1250)}^{\mathrm{obs}} \exp \left[l \left(\sigma_{(1225-1250)}\right.\right. \\
& \left.\left.-\sigma_{(1230-1300)}\right)\right]
\end{aligned}
$$

Applying also Eq. (4) to obtain $I_{(1550-1620)}^{\mathrm{obs}}$ from the 1540$1595 \AA$ unabsorbed spectrum, we find a $\mathrm{CH}_{4}$ effective column of $5 \times 10^{16} \mathrm{~cm}^{-2}$ and a color ratio of 2.26 . The total unabsorbed intensity of the $\mathrm{H}_{2}$ emission in the $1220-1740-\AA$ range may be estimated by multiplying the $I_{(1220-1740)}^{\text {ufit }} / I_{(1490-1540)}^{\text {ufit }}$ ratio of the synthetic spectrum by the total intensity of the second spectrum. We find a total unabsorbed intensity of 123 kilo-rayleighs which is in the range of values previously derived from HST spectra (Clarke et al. 1994, Trafton et al. 1994, 1998, Kim et al. 1997, Dols et al. 2000) (Table III).
TABLE III

Main Quantitative Results

$\begin{array}{cccc}\text { Central wavelength } & \text { View angle } & \text { Total } \mathrm{H}_{2} & \begin{array}{c}\text { Color ratio } \\ (\AA)\end{array} \\ (\mathrm{deg}) & (\mathrm{kR}) & (\text { observed })\end{array}$

\begin{tabular}{clrrr}
\hline Orbit 1 & & & \\
Absorbed & 1222 main oval & 54.2 & 123 & 2.26 \\
& 1222 polar cap & 54.2 & 130 & 2.83 \\
& 1222 limb & 54.2 & 150 & 1.45 \\
Unabsorbed & 1567 main oval & 54.2 & 123 & 2.26 \\
& 1567 polar cap & 54.2 & 130 & 2.83 \\
& 1567 limb & 54.2 & 150 & 1.45 \\
Orbit 2 & & & & \\
Absorbed & 1321 main oval & 46.6 & 125 & 5.93 \\
& 1321 pc & 46.6 & 75 & 7.43 \\
& 1321 limb & 46.6 & 105 & 2.59 \\
Unabsorbed & 1518 main oval & 46.6 & 125 & 5.93 \\
& 1518 polar cap & 46.6 & 75 & 7.43 \\
& 1518 limb & 46.6 & 105 & 2.59
\end{tabular}

The total $\mathrm{H}_{2}$ intensity can also be determined from the second STIS image by summing the pixel counts corresponding to the main oval, using the slit placed on the image. Based on the STIS absolute calibration, we obtain a total $\mathrm{H}_{2}$ intensity of 110 kilo-rayleighs, in very close agreement with the value deduced from the spectrum.

The best spectral fit is obtained by minimizing the $\chi^{2}$ between the synthetic and the observed spectra, as explained in Trafton et al. (1994) and Dols et al. (2000). In the present study, however, a major improvement to the Dols et al. (2000) method is brought by generating temperature and neutral composition vertical profiles consistently with the characteristics of electron energy and flux. The results of the fits are shown in Fig. 7 for the first (absorbed) spectrum. To remove the Ly $\alpha$ contribution, the best fit from the $\mathrm{H}_{2}$ lines obtained away from the broad wings of Ly $\alpha$ (Clarke et al. 1994), possibly due to excitation of fast hydrogen atoms as described by Bisikalo et al. (1996), is subtracted from the observed spectrum. The remaining Ly $\alpha$ contribution is then smoothed and subtracted from the observed spectrum, and the fitting procedure is applied to the resulting $\mathrm{H}_{2}$ spectrum. The agreement is very good and the $\mathrm{H}_{2}$ contribution is clearly identified in the vicinity of Ly $\alpha$. The best fit model used to generate the synthetic spectrum provides the values of the total $\mathrm{H}_{2}$ emission rate, the characteristic energy of the precipitating electrons, the emission peak altitude, and the hydrocarbon column densities above the peak. These values are summarized in Table IV. The derived energy flux of the hard electron energy component is $10 \mathrm{erg} \mathrm{cm}^{-2} \mathrm{~s}^{-1}$, and the mean energy $2 E_{0}$ is $80 \mathrm{keV}$.

This first component of the energy distribution is responsible for a relatively high value of the color ratio $(\sim 3)$, but since most of this energy is deposited at low altitudes, it does not contribute to the high average temperature that is mainly due to the second component. The second soft electron component is set to 
TABLE IV

Energy Fluxes and Characteristic Energies of the Maxwellian Components of the Energy Distributions for the Model Used in This Study

\begin{tabular}{cccccccc}
\hline $\begin{array}{c}\Phi_{0,1} \\
\left(\mathrm{erg} \mathrm{cm}^{-2} \mathrm{~s}^{-1}\right)\end{array}$ & $\begin{array}{c}E_{0,1} \\
(\mathrm{keV})\end{array}$ & $\begin{array}{c}\Phi_{0,2} \\
\left(\mathrm{erg} \mathrm{cm}^{-2} \mathrm{~s}^{-1}\right)\end{array}$ & $\begin{array}{c}E_{0,2} \\
(\mathrm{keV})\end{array}$ & $\begin{array}{c}\text { Color } \\
\text { ratio }\end{array}$ & $\begin{array}{c}Z_{\max } \\
(\mathrm{km})\end{array}$ & $\begin{array}{c}\mathrm{CH}_{4} \text { column } \\
\left(10^{17} \mathrm{~cm}^{-2}\right)\end{array}$ & $\begin{array}{c}\mathrm{C}_{2} \mathrm{H}_{2} \text { column } \\
\left(10^{16} \mathrm{~cm}^{-2}\right)\end{array}$ \\
\hline 10 & 40 & 5 & 0.1 & 2.1 & 222 & 4.1 & 0.12 \\
\hline
\end{tabular}

$5 \mathrm{erg} \mathrm{cm}^{-2} \mathrm{~s}^{-1}$. It gives rise to a high-altitude secondary peak in the volume emission rate which shifts the weight on the vertical profile of the temperature toward higher values and leads to an average temperature of $570 \mathrm{~K}$, at the expense of a slight decrease of the color ratio (2.8).

An analysis of the intensity distribution of the $\mathrm{H}_{2}$ lines was applied to the main oval, the polar cap, and the limb aurorae. Figure 4 shows that the overall shape of the absorbed spectra (1194-1250 $\AA$ ) is nearly the same for the three regions. At $\sim 1-\AA$ resolution, the influence of temperature is noticeable for almost half of the lines contained in the spectrum. As shown in Fig. 7, due to the limited signal-to-noise ratio, some line intensities suggest a high temperature while others indicate a low temperature. However, the behavior of the Werner (3-7) Q(3) line at $1231.95 \AA$, which is one of the features most sensitive to the temperature of the absorbed spectrum, suggests that the average temperature is the same in the three regions.

In the unabsorbed spectrum (1540-1595 $\AA$ ), the $\mathrm{H}_{2}$ Lyman continuum is clearly influenced by temperature (Abgrall et al. 1997). Figure 8 shows that the $1565-1585$ - $\AA$ region is brighter than the $1545-1560-\AA$ interval. The second interval increases with temperature relative to the first region. Also, the intensities of two features are very temperature-dependent. The first feature is a combination of two lines at 1585.43 and $1585.64 \AA$ : the $\mathrm{L}(2-9) \mathrm{P}(5)$ and $\mathrm{L}(6-12) \mathrm{P}(4)$. The second feature is a Lyman (6-12) $\mathrm{P}(5)$ line at $1587.42 \AA$. The model leading to the best fit is the same as that used for the absorbed spectrum: it gives the lowest $\chi^{2}$ and fits the 1585.43 and $1585.64 \AA$ lines well. The discrepancies observed between 1540 and $1560 \AA$ may be due to an underestimate of the Lyman continuum brightness. However, if the $\mathrm{H}_{2}$ temperature is higher, the continuum intensity in the $1540-1560-\AA$ domain increases and the relative line intensities are modified, resulting in an overall fit with a higher $\chi^{2}$.

Some tests were made to determine the temperature in the $1580-1590-\AA$ window, disregarding the other lines of the spectrum, and the results confirm the use of the same model as for the first orbit. The overall shape of the spectra in the three regions of the aurora is comparable and due to this similarity; the various temperature models used to fit the limb and the polar cap regions do not lead to any significant improvement of the overall $\chi^{2}$. Figure 9 illustrates this temperature effect and suggests that the temperatures of the main oval and polar cap regions are quite close. This result is consistent with the observed color ratios of the two regions which are also almost equal.
It is of interest to compare the rovibrational $\mathrm{H}_{2}$ temperatures, which may be deduced from the two STIS spectra using the thin slab approximation, that is, assuming that the emission arises from a region characterized by a single temperature. The temperature best fitting the unabsorbed spectrum of the main oval is about $410 \mathrm{~K}$. As expected, a slightly higher value of $550 \mathrm{~K}$ provides the best fit for the absorbed spectrum. This difference confirms that this emission originates from a warmer region located near and above the methane homopause. However, the same electron energy spectrum provides the best fit to both spectra since the attenuation by $\mathrm{CH}_{4}$ is internally accounted for by the model.

\section{IV.2. Second Orbit: August 13, 15:26-16:11}

Two spectra were taken close in time in the second orbit, following a FUV MAMA image. The same procedure was used to superimpose the projection of the $52 \times 0.5 \operatorname{arcsec}^{2}$ slit used for the spectra on the second image in order to derive a total auroral intensity. We again first concentrate on the spectra extracted from the main oval region. The short-wavelength spectrum shown in Fig. 11 is characterized by an $\mathrm{H}_{2}$ intensity of 1.5 kilo-rayleighs in the spectral window while the second spectrum has a brightness
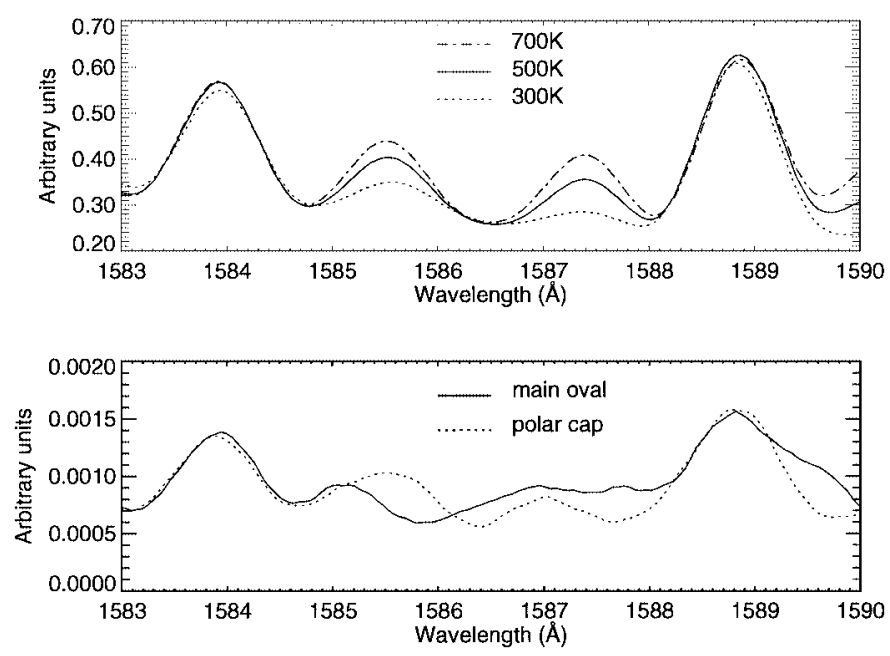

FIG. 9. Effect of temperature in the 1583-1590- $\AA$ window. The L(2-9) $\mathrm{P}(5)$ at $1585.43 \AA$, the $\mathrm{L}(6-12) \mathrm{P}(4)$ at $1585.64 \AA$, and the $\mathrm{L}(6-12) \mathrm{P}(5)$ at $1587.42 \AA$ increase with $T$ (top). This effect is not obvious in the polar cap and main oval regions of the data, suggesting a temperature of the same order (bottom). 
of 10.7 kilo-rayleighs. The total $\mathrm{H}_{2}$ intensity calculated from the second (unabsorbed) spectrum is 125 kilo-rayleighs. The total intensity estimated from the projection of the slit on the second image of the orbit is 120 kilo-rayleighs, again in excellent agreement with the spectrally derived value.

The same general method is used to determine the model parameters that best fit the spectra. The absorbed spectrum is more difficult to use owing to its low signal-to-noise ratio and the subtraction of the dark component. This spectrum (Fig. 11) contains two lines whose intensity is markedly temperature-dependent. The first one is a blend of the $\mathrm{L}(0-4) \mathrm{R}(3)$ at $1337.47 \AA$ and the $\mathrm{L}(5-6) \mathrm{R}(3)$ lines at $1337.85 \AA$. The second feature is the $\mathrm{L}(0-4)$ $\mathrm{P}(2)$ line at $1338.57 \AA$. The relative brightness of the lines of the main oval fit is good and indicates that the mean auroral temperature in the main oval region is $\sim 540 \mathrm{~K}$. The fit does not match the 1336-1340- $\AA$ region well, because the fitting procedure minimizes the $\chi^{2}$ and take into account the global spectral window. The 1295-1330-Å region shows significant discrepancies that can partly be ascribed to the residual presence of the terrestrial OI triplet at $1302.17,1304.86$, and $1306.03 \AA$ in the observed spectrum following subtraction of the sky background contribution. The presence of the $1304 \AA$ triplet in the jovian auroral spectrum has been a matter of controversy since Waite et al. (1998) observed a weak feature in jovian auroral spectra obtained with the spectrograph on board the International Ultraviolet Explorer (IUE) satellite. Trafton et al. (1998) analyzed a spectrum obtained between 1282 and $1318 \AA$ at 0.5 - $\AA$ resolution with the Goddard High Resolution Spectrometer (GHRS). They set an upper limit of about 40 rayleighs on the brightness of the triplet. They also indicate that a broad feature is expected from precipitation of $\mathrm{O}^{+}$ions gyrating along the magnetic field lines rather than discrete lines. In the present spectrum, the presence of the STIS detector noise in the 1300 - $\AA$ region, combined with the geocoronal contamination, prevents setting a reasonable upper limit on the $1304-\AA$ emission possibly associated with oxygen ion precipitation.

The unabsorbed spectrum of the main oval shown in Fig. 12 also includes a feature very sensitive to temperature which is the blend of two lines: the $\mathrm{L}(1-7) \mathrm{P}(5)$ line at $1504.76 \AA$ and the $\mathrm{L}(10-13) \mathrm{P}(2)$ at $1504.93 \AA$ A. Figure 10 illustrates this temperature dependence for a synthetic spectrum emitted by a homogeneous layer of $\mathrm{H}_{2}$ at various temperatures. It also shows a comparison between the main oval and the polar cap spectrum. A strong $\mathrm{C}_{2} \mathrm{H}_{2}$ absorption peak is observed near $1519.4 \AA$, and a series of fits with various atmosphere models were calculated. The main difficulty is to reconcile a model with an appropriate $\mathrm{H}_{2}$ temperature and $\mathrm{C}_{2} \mathrm{H}_{2}$ column. The best compromise is case A: a model with a higher mean temperature generates too much absorption, and a model with the correct amount of absorption is too cold compared to the $\mathrm{H}_{2}$ best fit. As seen in Fig. 10, a careful study of the temperature-dependent lines and the partially absorbed line at $1519.4 \AA$ is sufficient to qualitatively estimate the temperature of the three auroral regions. The $L(1-7)$ $\mathrm{P}(5)$ line is the brightest for the limb while the $1519.4 \AA$ line is
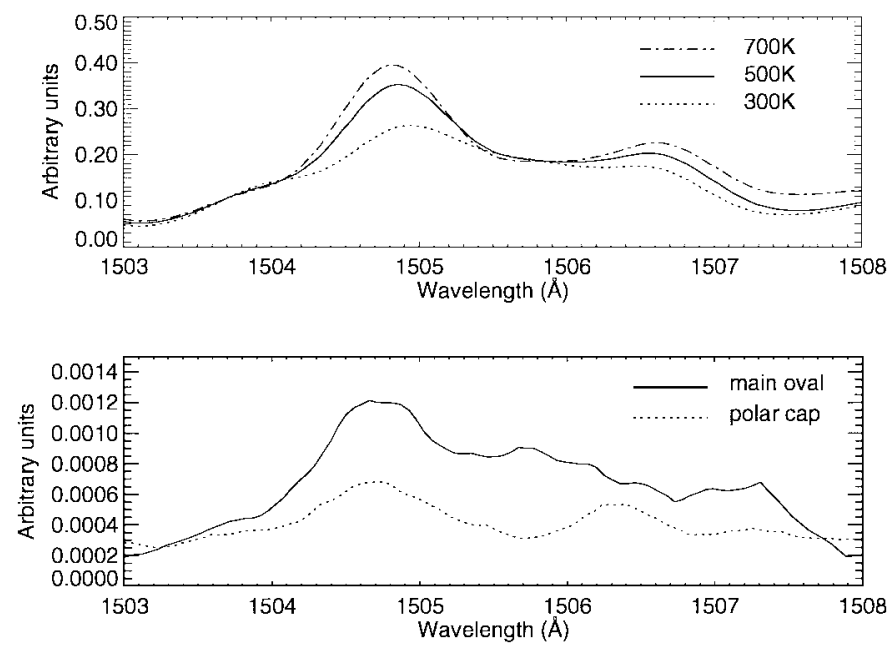

FIG. 10. Effect of temperature in the 1503-1508- $\AA$ window. As for Fig. 9, the $\mathrm{L}(1-7) \mathrm{P}(5)$ at $1504.76 \AA$ and the $\mathrm{L}(10-13) \mathrm{P}(2)$ at $1504.93 \AA$ increase with $T$ (top). The temperature effect on the data suggests a higher temperature for the main oval, compared with the polar cap.

also the highest. The polar cap has the lowest $\mathrm{L}(1-7) \mathrm{P}(5)$ line, and the line at $1519.4 \AA$ is the most absorbed if compared to the adjacent lines. This conclusion is also confirmed by the color ratio, which varies from 2.6 for the limb to 7.4 in the polar cap.

As for the first orbit, the first spectrum is partially absorbed by methane, while the second is not. Using the same method as for the first set of observations, we obtain an effective $\mathrm{CH}_{4}$ column of $6.2 \times 10^{17} \mathrm{~cm}^{-2}$ and a large color ratio of 5.9 in the main oval. The fit of the absorbed spectrum is shown in Fig. 11. The longer wavelength spectrum and its best fit using the same model are shown in Fig. 12. The $\mathrm{C}_{2} \mathrm{H}_{2}$ column deduced from the model is $1.2 \times 10^{15} \mathrm{~cm}^{-2}$, in the range of values deduced from HST-GHRS G140L spectra by Dols et al. (2000).

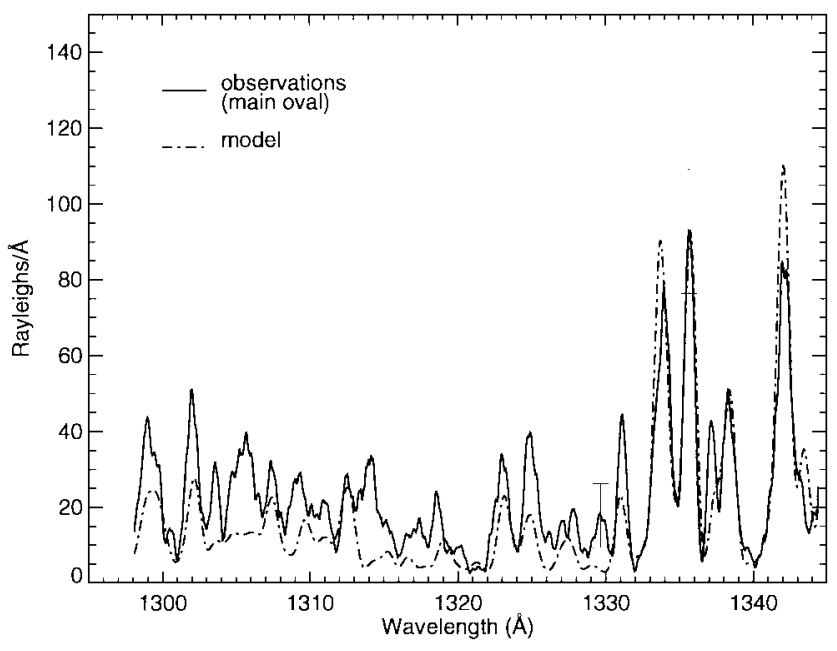

FIG. 11. Second short-wavelength spectrum and comparison with the best model fit. 


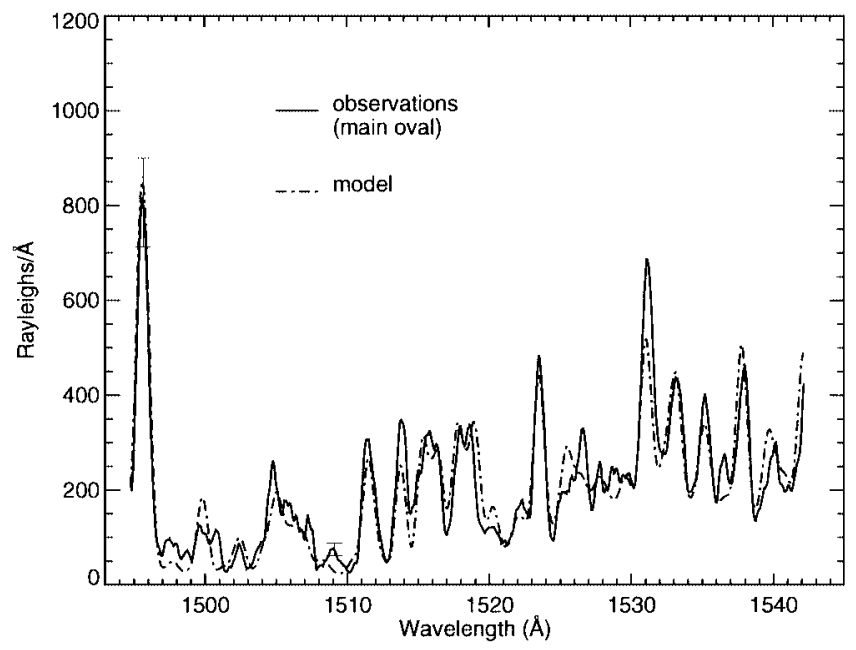

FIG. 12. Second long-wavelength spectrum and comparison with the best model fit.

\section{CONCLUSIONS}

Spatially resolved FUV spectroscopy with HSI-STIS has been used to investigate the characteristics of the jovian aurora. The brightnesses derived from the STIS spectra and images converge to a value of $\sim 120$ kilo-rayleighs of $\mathrm{H}_{2}$ emission, in agreement with previous studies based on other instruments. This stability of the brightness of the main oval aurora significantly contrasts with that of the Earth aurora, which is highly dependent on the conditions prevailing in the upstream solar wind.

Distinct color ratios and methane columns are associated with the different auroral regions observed across the STIS slit. Although the polar cap and main oval emissions have nearly equal values for the first spectral pair, the values obtained for the second orbit are significantly higher, with slightly more absorption in the polar cap. It is thus suggested that the auroral mean electron energy varies in time and space.

The $\mathrm{H}_{2}$ temperature derived from the overall intensity distribution of the $\mathrm{H}_{2}$ lines is about $540 \mathrm{~K}$. This determination is limited by the STIS sensitivity, in particular when data from spatially restricted regions are extracted. However, temperaturesensitive lines have been used to determine possible temperature variations across the north polar region. The first pair of spectra shows no significant temperature difference between the main oval and the polar cap, as expected from the small difference in color ratios between the two regions. In contrast, the second spectral pair indicates a lower temperature $(\sim 300 \mathrm{~K})$ in the polar cap, in agreement with the larger methane absorption observed in this region.

The model globally best fitting the observed $\mathrm{H}_{2}$ intensity distribution has a mean energy of $\sim 80 \mathrm{keV}$, a value on the higher side of previously determined energies. Other studies have yielded values of the same order. More accurate conclusions concerning the spatial variation along the STIS slit are hampered by the lack of simultaneous STIS spectroscopy in the
G140M mode. Spectroscopy with the G140L mode, covering the complete FUV range could advantageously be used for this purpose. However, it does not offer the spectral resolution necessary to determine $\mathrm{H}_{2}$ rovibrational temperatures.

\section{ACKNOWLEDGMENTS}

J.C.G. and D.G. are supported by the Belgian Foundation for Scientific Research (FNRS). This work is based on observations with the NASA/ESA Hubble Space Telescope, obtained at the Space Telescope Science Institute (STScI), which is operated by AURA, Inc. for NASA under Contract NAS5-26555. The research was funded by the PRODEX ESA program supported by the Belgian Federal Office for Scientific, Technical, and Cultural Affairs-Prime Minister's services and Grant GO-8171.99-A from the STScI to the University of Michigan.

\section{REFERENCES}

Abgrall, H., E. Roueff, X. Liu, and D. E. Shemansky 1997. The emission continuum of electron-excited molecular hydrogen. Astrophys. J. 481, 557566.

Ajello, J. M., D. E. Shemansky, W. Pryor, K. Tobiska, C. Hord, S. Stephens, I. Stewart, J. Clarke, K. Simmons, W. McClintock, C. Barth, J. Gebben, D. Miller, and B. Sandel 1998. Galileo orbiter ultraviolet observations of Jupiter aurora, J. Geophys. Res. 103, 20,125-20,148.

Ajello, J. M., D. E. Shemansky, W. R. Pryor, A. I. Stewart, K. E. Simmons, T. Majeed, J. H. Waite, R. G. Gladstone, and D. Grodent 2001. Spectroscopic evidence for high-altitude aurora at Jupiter from Galileo Extreme Ultraviolet Spectrometer and Hopkins Ultraviolet Telescope observations. J. Geophys. Res. 523, 151-171.

Bisikalo, D. V., V. I. Shematovich, J. C. Gérard, R. G. Gladstone, and J. H. Waite 1996. The distribution of hot hydrogen atoms produced by electron and proton precipitation in the jovian aurora. J. Geophys. Res. 101, 21,157-21,168.

Clarke, J. T., L. Ben Jaffel, A. Vidal-Madjar, G. R. Gladstone, J. H. Waite, Jr., R. Prangé, J.-C. Gérard, J. Ajello, and G. James 1994. Hubble Space Telescope Goddard High-Resolution Spectrograph $\mathrm{H}_{2}$ rotational spectra of Jupiter's aurora. Astrophys. J. 430, L73-76.

Dols, V., J. C. Gérard, J. T. Clarke, J. Gustin, and D. Grodent 2000. Diagnostics of the jovian aurora deduced from ultraviolet spectroscopy: Model and GHRS observations. Icarus 147, 251-266.

Drossart, P., J.-P. Maillard, J. Caldwell, and J. Rosenqvist 1993. Line-resolved spectroscopy of the Jovian $\mathrm{H}_{3}^{+}$auroral emission at 3.5 micrometers. Astrophys. J. 402, L25-28.

Gérard, J.-C., D. Grodent, V. Dols, and J. H. Waite, Jr. 1998. The longitudinal variation of the color ratio of the jovian ultraviolet aurora: A geometric effect? Geophys. Res. Lett. 25, 1601-1604.

Gladstone, G. R., M. Allen, and Y. L. Yung 1996. Hydrocarbon photochemistry in the upper atmosphere of Jupiter. Icarus 119, 1-52.

Grodent, D., J. H. Waite, and J. C. Gérard 2001. A self-consistent model of the jovian auroral thermal structure, 2001. J. Geophys. Res. 106, 12,933-12,952.

Harris, W., J. T. Clarke, M. A. McGrath, and G. E. Ballester 1996. Analysis of jovian auroral H Ly- $\alpha$ emission (1981-1991). Icarus 124, 350-365.

Kim, Y. H., J. L. Fox, and J. J. Caldwell 1997. Temperatures and altitudes of Jupiter's ultraviolet aurora inferred from GHRS observations with the Hubble Space Telescope. Icarus 128, 189-201.

Lam, H. A., N. Achilleos, S. Miller, J. Tennyson, L. M. Trafton, T. R. Geballe, and G. E. Ballester 1997. A baseline spectroscopic study of the infrared auroras of Jupiter. Icarus 127, 379-393.

Landsman, W. 1998. Characteristics of the FUV-MAMA Dark Rate. STSCI Report. http://hires. gsfc. nasa. gov/stis/postcal/quick_reports/r057/r057.html. 
Livengood, T. A., D. F. Strobel, and H. W. Moos 1990. Long-term study of longitudinal dependence in primary particle precipitation in the north jovian aurora. J. Geophys. Res. 95, 10,375-10,383.

Perry, J. J., Y. H. Kim, J. L. Fox, and H. S. Porter 1999. Chemistry of the jovian auroral atmosphere. J. Geophys. Res. 104, 16,541-16,565.

Pryor, W. R., A. I. F. Stewart, K. E. Simmons, W. E. McClintock, W. Sweet, J. M. Ajello, G. K. James, W. K. Tobiska, G. R. Gladstone, D. E. Shemansky, A. P. Ingersoll, A. R. Vasavada, and J. T. Clarke 2001. Jupiter's UV aurora on Galileo Orbit G7. Icarus, in press.

Seiff, A., D. B. Kirk, T. C. D. Knight, R. E. Young, J. D. Mihalov, L. A. Young, F. S. Milos, G. Schubert, R. C. Blanchard, and D. Atkinson 1998. Thermal structure of Jupiter's atmosphere near the edge of a 5- $\mu \mathrm{m}$ hot spot in the north equatorial belt. J. Geophys. Res. 103, 22,857-22,890.

Trafton, L. M., J. C. Gérard, G. Munhoven, and J. H. Waite, Jr. 1994. Highresolution spectra of Jupiter's northern auroral ultraviolet emission with the Hubble Space Telescope. Astrophys. J. 421, 816-827.
Trafton, L. M., V. Dols, J.-C. Gérard, J. H. Waite, Jr., G. R. Gladstone, and G. Munhoven 1998. HST spectra of the jovian ultraviolet aurora: Search for heavy ion precipitation. Astrophys. J. 507, 955-967.

Vasavada, A. R., A. H. Bouchez, A. P. Ingersoll, B. Little, C. D. Anger, and the Galileo SSI Team 1999. Jupiter's visible aurora and Io footprint. J. Geophys. Res. 104, 27,133-27,142.

Waite, J. H., J. T. Clarke, T. E. Cravens, and C. M. Hammond 1998. The jovian aurora: Electron or ion precipitation? J. Geophys. Res. 93, 72447250 .

Wolven, B. C., and P. Feldman 1998. Self-absorption by vibrationally excited $\mathrm{H}_{2}$ in the Astro-2 Hopkins Ultraviolet Telescope spectrum of the jovian aurora. Geophys. Res. Lett. 25, 1537-1540.

Yung, Y. L., G. R. Gladstone, K. M. Chang, J. M. Ajello, and S. K. Srivastava 1982. $\mathrm{H}_{2}$ fluorescence spectrum from 1200 to 1700 by electron impact: Laboratory study and application to jovian aurora. Astrophys. J. 254, L65-69. 